Research Article

\title{
Flow Process Development and Optimization of A Suzuki-Miyaura Cross Coupling Reaction using Response Surface Methodology
}

\author{
Girish Basavaraju ${ }^{1,2, *}$, Ravishankar Rajanna ${ }^{1}$ \\ ${ }^{1}$ Department of Chemical Engineering, Dayananda Sagar College of Engineering, Bengaluru, India. \\ ${ }^{2}$ Syngene International Ltd, Bengaluru, India.
}

Received: 22 ${ }^{\text {nd }}$ June 2020; Revised: 24th July 2020; Accepted: 25th July 2020;

Available online: 15th August 2020; Published regularly: December 2020

\begin{abstract}
A custom-made tubular flow reactor was utilized to develop a mathematical model and optimize the Suzuki-Miyaura cross coupling reaction. In this study, the experimentation was designed and executed through the statistical design of experiments (DoE) approach via response surface methodology. The effect of molar ratios of phenylboronic acid (1) and 4-bromophenol (2), temperature, the catalyst tetrakis(triphenylphosphine)palladium, and equivalence of aqueous tripotassium phosphate was studied in detail. The flow reactor profile was in good agreement with batch conditions and significant improvements to the overall reaction time and selectivity towards desired [1-1-biphenyl]-4-ol (3) was achieved. The Suzuki coupling reaction in batch condition would take on an average of 4 to 6 hours to complete, which was effectively accomplished in 60 to 70 minutes in this tubular reactor setup and could be operated continuously. The reaction model is in good agreement with the reaction conditions. Copyright (C) 2020 BCREC Group. All rights reserved
\end{abstract}

Keywords: Continuous flow chemistry; Suzuki coupling reaction; Customized flow reactor; Tubular reactor; Design of experiments (DoE)

How to Cite: Basavaraju, G., Rajanna, R. (2020). Flow Process Development and Optimization of A Suzuki-Miyaura Cross Coupling Reaction using Response Surface Methodology. Bulletin of Chemical Reaction Engineering \& Catalysis, 15(3), 604-616 (doi:10.9767/bcrec.15.3.8229.604-616)

Permalink/DOI: https://doi.org/10.9767/bcrec.15.3.8229.604-616

\section{Introduction}

In recent years, the $\mathrm{C}-\mathrm{C}$ bond formation through metal-catalyzed coupling reactions was explored in various ways. As this reaction being regularly used across chemical transformations in organic chemistry, involves coupling partners such as boronic acid and an organohalide [1-5]. This chemistry under homogeneous and heterogeneous conditions with various metal compositions enable the synthesis of complex and func-

\footnotetext{
* Corresponding Author.

E-mail: girish.basavaraju@gmail.com (G. Basavaraju); Telp: +91-9980455822
}

tionalized organic molecules [3-4]. This process is frequently utilized in the synthesis of substituted polyolefins, biphenyls, and styrenes. To name a few, metal-catalyzed coupling reactions such as Suzuki, Sonogashira, Heck, BuckwaldHartwig, Negishi, and Kumuda have been demonstrated over the years $[7-9,14-16]$. These reactions are less toxic, inexpensive, easily prepared, and environment-friendly over other organostannane and organozinc compounds [9-10].

The Suzuki coupling mechanism follows a general catalytic cycle including three primary steps namely, oxidative addition, transmetalation, and reductive elimination [14]. The 
hybrid and more effective processes meeting few green chemistries and sustainable principles are being worked out in academics and industrial setups [9-10]. Since the Suzuki Miyuara cross-coupling finds application in many fields including industrial chemicals and pharmaceuticals, developing various process technologies and optimization techniques find their importance. Out of the several advancements and approaches being tested, continuous flow processes are one among the technology that offers significant value to the product life cycle [11-14].

Continuous process development has witnessed a significant change in the last decade, and there is a spike in the number of publications across the industry sector, especially pharmaceutical and fine chemicals [17]. This flow technology is very familiar in the commodity chemicals and very recently being adapted to research and development and offers reproducibility, superior heat and mass transfer, low footprint, fast and effective mixing high reactor throughput, safety, waste minimization, energy efficiency and environmental impact [18-24]. The main advantages of adapting the flow processes at the research level would be considering the future of the process and product. The laboratory developed processes could be scaled to pilot and commercial scales with minimal re- sources, without considerably modifying the reaction conditions [25-27].

On the other side, various statistical methods enable the experimenter to develop and optimize the processes under fast-track mode. A simple and effective approach would be the design of experiments (DoE), which has gained importance across researchers to develop their experimental plan [28-33]. This approach offers minimum experimentation and enables the experimenter to establish a rational and relationship of factors and their interactions to conclude a meaningful outcome. The overall process provides proof to discover the unknown effect, determine or test the hypothesis, and prove with the mathematical numbers. In recent times, these methodologies were utilized in large numbers across research and development and manufacturing operations to gather maximum data with minimum efforts [34-39].

In the literature, the Suzuki coupling reaction was not well studied under flow conditions and minimal or no efforts towards process optimization exercise including mathematical model development. In short, the objective of the study is to develop a successful continuous flow process technology to transform an existing Suzuki coupling batch process to continuous and optimize the reaction conditions using response surface methodology.

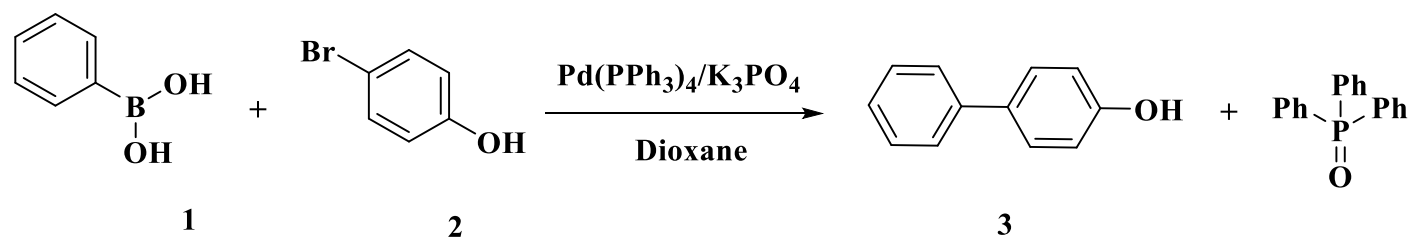

Impurity

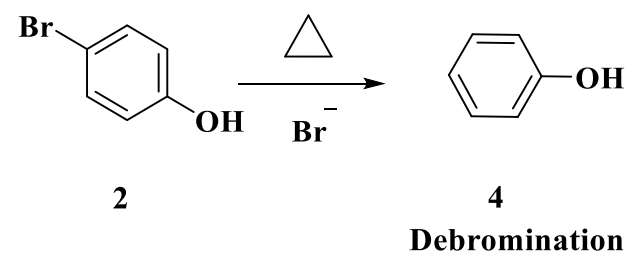

Impurity

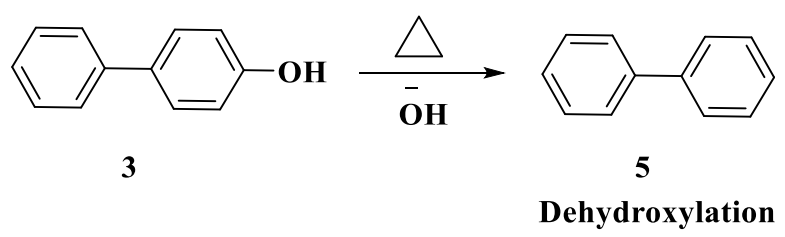

Scheme 1. Reaction scheme and mechanism of Suzuki coupling reaction. 
In the current study, a custom-made flow reactor was utilized to develop the model and optimize the reaction condition of a homogeneous Suzuki coupling reaction (Scheme 1). The synthesis of [1-1-biphenyl]-4-ol (3) was conducted using phenylboronic acid (1) and 4bromophenol (2) in presence of catalyst tetrakis(triphenylphosphine)palladium, aqueous tripotassium phosphate, and 1,4-dioxane as a solvent. The learnings from the batch process were transformed to develop, optimize, and intensify under flow conditions using a customized tubular flow reactor. A statistical DoE approach was used to design an experimental plan, model, and optimize the reaction under flow conditions. The benefits and opportunities were summarized.

\section{Material and Methods}

The chemicals phenylboronic acid (Merck, 95\%) and 4-bromophenol (Merck, 96\%), catalyst tetrakis(triphenylphosphine)palladium (Merck, $>99 \%$ ), base tripotassium phosphate (Merck, $\geq$ $98 \%$ ), and solvent 1,4-dioxane (Spectrochem, > $99.5 \%)$.

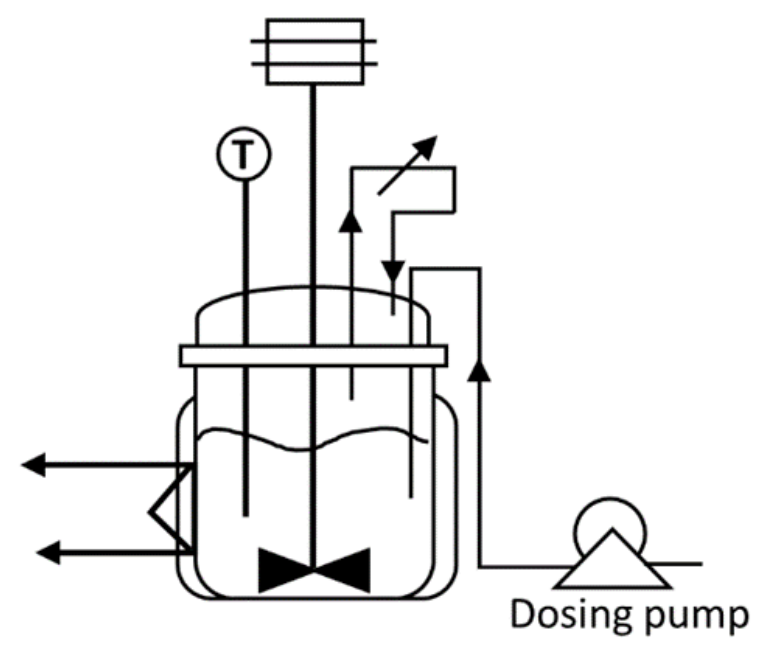

Figure 1. Schematic of the batch reactor.

\subsection{Setup of Batch Reactor}

A schematic representation of a batch reactor setup is shown in Figure 1. All the batch experiments were performed in a jacketed reactor setup equipped with a $100 \mathrm{~mL}$ reactor fitted with a coiled condenser. The pitch blade turbine was used as an agitator for conducting experiments. The head of the reactor had 4 ports, of which one was used for a temperature probe (T), another for the agitator shaft, a third for condenser along with nitrogen vent and fourth was for dosing reagents. The detailed experimental procedure is available in the supporting information.

2.2 Continuous Flow Process Design of Tubular Reactor Setup

The tubular flow reactor was fabricated using perfluroalcoxy alkane (PFA) tubes with internal diameter $3.175 \mathrm{~mm}$ (supplied by Swagelok, Bengaluru) coiled with a definite volume of $30 \mathrm{~mL}$ rolled into a disc form, connected with a T-joint and a static mixer (supplied by Cole-Parmer, Bengaluru) and immersed into a bath circulator. Two pumps A and B (QG50, supplied by FMI, Inc, Chennai) were used to pump the reaction mixture at a definite flow rate. The open bath circulator (supplied by Huber, Germany) was used to control the temperature of the flow reactor. The schematic of the experimental setup is shown in Figure 2. A clean water test was conducted in this reactor to set the standard time for reaching equilibrium temperature. Around 15 min were allowed as a buffer temperature for each temperature change so that the temperature in the reactor is attained like the bath temperature. The variation was around $1.2{ }^{\circ} \mathrm{C}$ in a tubular flow reactor, which was corrected internally while running experiments.

To a $100 \mathrm{~mL}$ conical flask-A, prepared aqueous tripotassium phosphate added phenylboronic acid (1) and 1,4-dioxane. To another

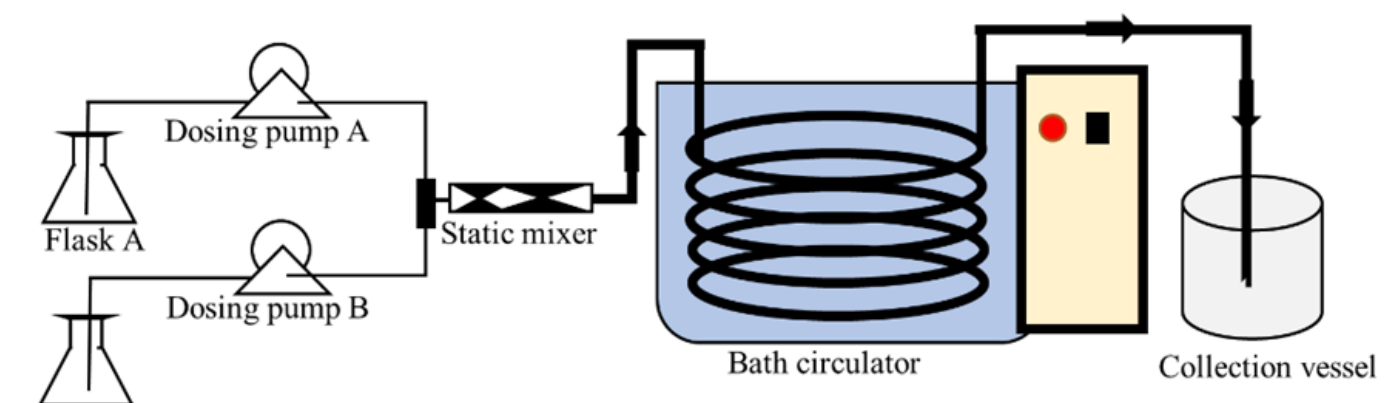

Tubular Reactor

Figure 2. Schematic of the tubular flow reactor.

Copyright $\odot$ 2020, BCREC, ISSN 1978-2993 
conical flask-B, charged accurately weighed 4bromophenol (2), 1,4-dioxane, and tetrakis followed by purging with nitrogen for 2 to 5 minutes and sealed the flask with rubber cork. The two metering pumps $\mathrm{A}$ and $\mathrm{B}$ were precalibrated based on the required flow rate and the residence time. The two conical flasks containing stock solutions were connected to pump A and pump B respectively. The discharge ends of these pumps were connected to a T-joint and a static mixer followed by a tubular flow reactor, which is externally heated through the circulator. The temperature of the circulator bath was maintained through the thermostat. The desired temperature was set on the thermostat, after achieving the temperature, simultaneously both the pumps were switched 'ON' and allowed reagents to pass through and react based on the pre-defined residence time. The flow rates of each pump were in the range of 0.275 and $0.224 \mathrm{~mL} / \mathrm{min}$, respectively. The residence time of around 60 minutes was kept constant for all the runs. The experiments were performed at three different temperatures, such as: 60,80 , and $100{ }^{\circ} \mathrm{C}$. The molar ratios of 1 , 1.15 , and 1.3 of phenylboronic acid (1) and 4bromophenol (2), were used with varying catalyst tetrakis(triphenylphosphine)palladium equivalence and base tripotassium phosphate equivalence respectively. The experiments were executed as per the run order (Table 1). The output of the reactor was connected to a collection vessel. The samples were drawn directly from the tube end and analyzed through offline HPLC. The total reaction mixture was worked out at the end of each experiment to get a final product as solid. The reactor was rinsed with process solvent to ensure no residue is available for the next experiment.

\subsection{Analytical Method Development}

The reaction was performed at $1.0 \mathrm{~g}$ scale like the regular experiment, after completion of the reaction, the mixture was diluted with water and extracted in ethyl acetate. The organic layer was separated and washed with the brine solution. A known amount of sodium sulfate was added and stirred for a couple of minutes to remove the excess moisture content and the solution was filtered using Buchner funnel. The filtrate was concentrated using rotavapor and the product was dried and submitted for HPLC for purity and NMR. Further purification of the product was done through prep-HPLC to achieve samples for analytical standards. The synthesized compound [1-1-biphenyl]-4-ol (3) along with phenylboronic acid (1) and 4- bromophenol (2) were used to develop an analytical method through HPLC. Here we used area normalization method for our initial HPLC analysis, which we believe fairly a good start, further weight percentage method will add more details to the quantification. The experimental samples were analyzed using the HPLC method and tabulate for data analysis. Detailed analytical method information along with column details provided in the supporting information.

\subsection{Design of Experiments}

The design of experiments (DoE) approach was used to design the experimental plan for the tubular reactor setup. A response surface methodology (RSM) was employed for designing the experimental plan. Four factors, such as: the molar ratio of phenylboronic acid (1) and 4-bromophenol (2), catalyst tetrakis(triphenylphosphine)palladium equivalence, reaction temperature, the equivalence of base tripotassium phosphate, were considered. The solvent 1,4-Dioxane was kept constant at 30 volumes based on the batch size. The experimental design pattern was created using Design-Expert software, version 11.0. The design was a central composite orthogonal design with 3 center points, limited to main effects and interaction effects with a total of 21 experiments. These experiments were planned to investigate each effect of the parameters separately and in pairs. Every individual parameter was assigned with a high and a low value based on the observations from the screening experiments. To simplify the computations and analysis, each factor was codified as: $(-1),(+1)$, and ' 0 ' for minimum, maximum, and as center point average values, respectively.

The experiments were executed as per the run order of the design pattern, which was already randomized to minimize noise from the software itself. To estimate the curvature effects and deviations in experimental reproducibility, if any, three center points were included in the design pattern. Based on our understanding and confidence, the reaction inprocess control (IPC) data was used effectively to develop, model, and optimize the reaction conditions and presented in this study. The responses, such as: yield and the controlling factors, were modeled and optimized using the RSM.

The value of star point, $\alpha$, for orthogonality depends on the number of points in the factorial portion of the design which is given in Equation (1). 


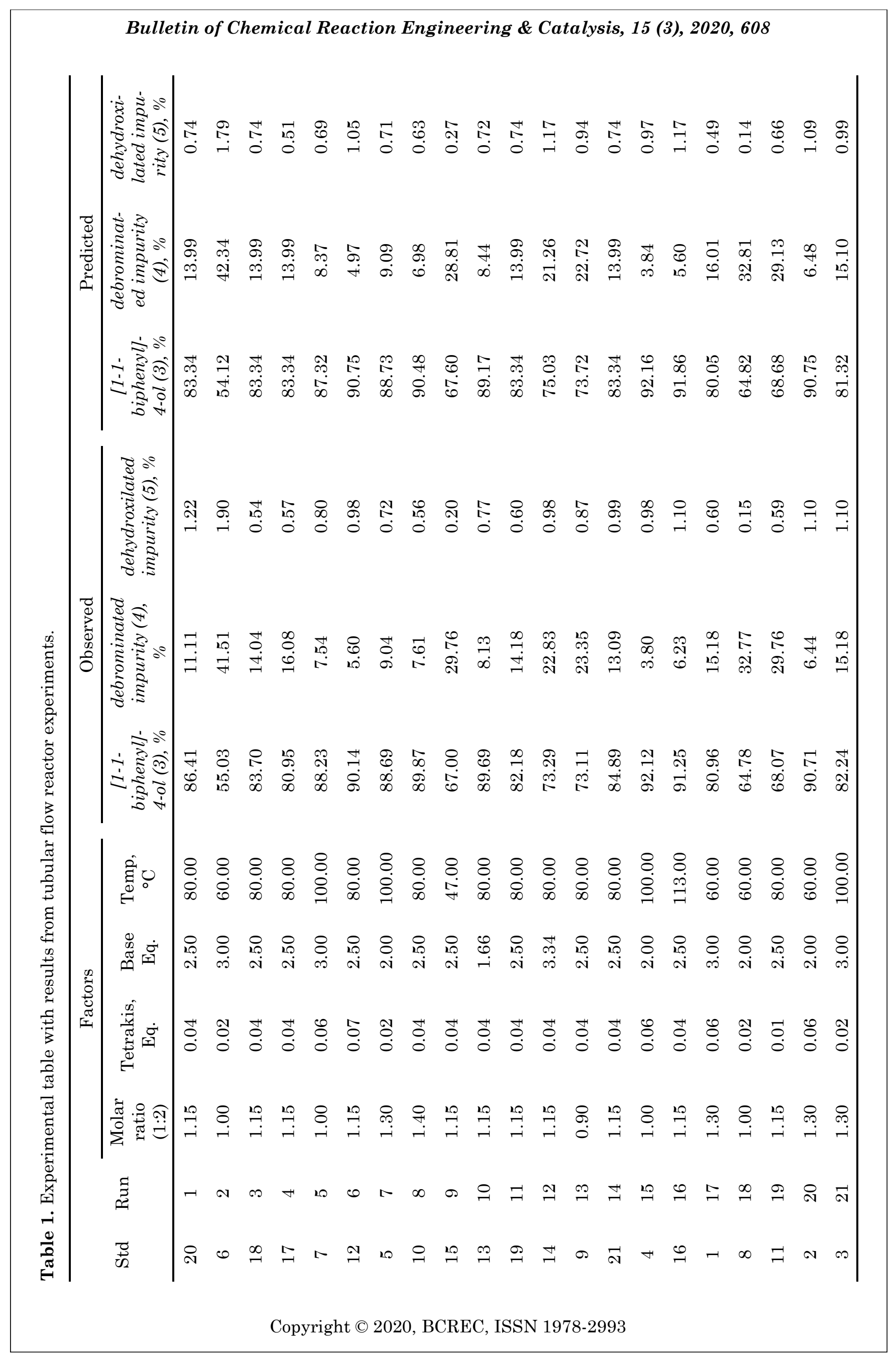




$$
\alpha=\left\{\left[\left(n_{c}+n_{s}+n_{0}\right)^{1 / 2}-n_{c}^{1 / 2}\right]^{2} * n_{c} / 4\right\}^{1 / 4}
$$

where $n_{c}$ is the number of points in the cube portion of the design, $n_{s}$ is the number of star points, and $n_{0}$ is the number of center points. Four variables or factors such as the molar ratio $\left(X_{1}\right)$ of phenylboronic acid (1) and 4b r o mophenol ( 2 ), c a t a ly s t tetrakis(triphenylphosphine)palladium $\left(X_{2}\right)$ equivalence, reaction temperature $\left(X_{3}\right)$, the equivalence of base tripotassium phosphate $\left(X_{4}\right)$ were considered. The ranges of independent variables were chosen based on the conditions from the variables screened before optimization. The ranges and levels used in the experimental work are given in Table 1 . In the experimental design, all variables are coded for statistical calculation according to Equation (2).

$$
x_{i}=\frac{\alpha\left[2 X_{i}-\left(X_{\max }+X_{\min }\right)\right]}{X_{\max }-X_{\min }}
$$

where $x_{i}$ is the dimensionless coded value of the $\mathrm{i}^{\text {th }}$ variable, $X_{i}$ is the natural value of the $\mathrm{i}^{\text {th }}$ variable, $X_{\max }$ and $X_{\min }$ are the highest and the lowest limit of the $i^{\text {th }}$ variable respectively. The responses and the corresponding factors are modeled and optimized using the response surface methodology (RSM) [40].

A quadratic polynomial equation was developed to predict the response as a function of input variables and their interactions. The equations for 1-biphenyl]-4-ol (3), debrominated impurity (4), and dehydroxilated impurity (5) were established. In general, the response for the quadratic polynomials is described in Equation (3).

$$
Y=\beta_{0}+\sum_{j=1}^{2} \beta_{j} X_{j}+\sum_{j=1}^{2} \beta_{j j} X_{j}^{2}+\sum_{i<j} \beta_{i j} X_{i} X_{j}
$$

In this equation, $Y$ is the predicted response, $\beta_{0}$ is the intercept coefficient, $\beta_{j}$ are the linear terms, $\beta_{j j}$ are the squared terms, $\beta_{i j}$ are the interaction terms, and $X_{i}$ and $X_{j}$ represent the independent variables. Three-dimensional response surfaces and contour plots were used for analyzing the experimental output concerning inputs. Coefficients of the models were estimated using multiple regression analysis. The fit quality of the model was judged from their coefficients of correlation and determination. The accuracy and each model were also checked with the analysis of variance (ANOVA) using F-test, p-value, and represented.

\section{Results and Discussions}

The synthesis of the compound [1-1biphenyl]-4-ol (3) was investigated as a representative procedure for the synthesis of the $\mathrm{Su}$ zuki coupling reaction (Scheme 1). Screening experiments were performed at 100 to $250 \mathrm{mg}$ scale under batch conditions to understand the reaction behavior and factors affecting the reaction profile. The objective of this work was more centered and restricted to continuous flow process development with the data obtained from the batch experiments.

Batch experiments were performed at $1.0 \mathrm{~g}$ scale with temperatures 60 and $100{ }^{\circ} \mathrm{C}$. The IPC samples were drawn at every 60 minutes and analysed through offline HPLC to estimate the conversions and reaction profile. In the end, the reaction mass was worked up to isolate as crude solid. The solids were further purified to support analytical method development. The first hour IPC showed a conversion of about 25 to $30 \%$ with a selectivity of 18 to $23 \%$ towards compound [1-1-biphenyl]-4-ol (3) respectively. The 100 and $60{ }^{\circ} \mathrm{C}$ experiments under standard baseline conditions completed in about 3 and 5 hours respectively. The reaction behavior was in good agreement with the screening experiments. The reaction conditions with higher temperatures were quick when compared to lower temperature conditions. The reaction conversion was more than $98 \%$ and selectivity towards compound [1-1-biphenyl]-4-ol (3) was around 70 to $80 \%$ consistently. Overall, the reaction rate increases by 1 to 2 -fold with a 60 to $100{ }^{\circ} \mathrm{C}$ increase in temperature. The impurities (4) and (5) were observed reasonably at higher levels in the range of $15 \%$ and $3 \%$ respectively. The final yields of [1-1-biphenyl]-4ol (3) were in the range of 80 to $85 \%$. We believe the yields could be improved further with higher batch sizes, as handling losses could be minimized. These batch experiments provided us a good understanding of reaction behavior and kinetics to design our experimental plan for continuous flow process using a tubular reactor setup.

\subsection{Discussion on Tubular Flow Reactor}

The experimental result was tabulated in the design expert template to identify and analyze the significant factors and their effects in the formation of [1-1-biphenyl]-4-ol (3), (4), and (5). Contour and surface plots for compound [11-biphenyl]-4-ol (3), dibrominated impurity (4), and dehydroxilated impurity (5) were obtained from DoE analysis were represented in Figures 
3 to 11. Based on the analysis, it was found that the compound [1-1-biphenyl]-4-ol (3) increases with an increase in temperature, catalyst tetrakis(triphenylphosphine)palladium equivalence, and the molar ratio of phenylboronic acid (1) and 4-bromophenol (2) and has an inverse impact with an increase in base tripotassium phosphate equivalence. On the other side, the level of debrominated impurity (4) was found to increase when, the base tripotassium phosphate equivalence was loaded

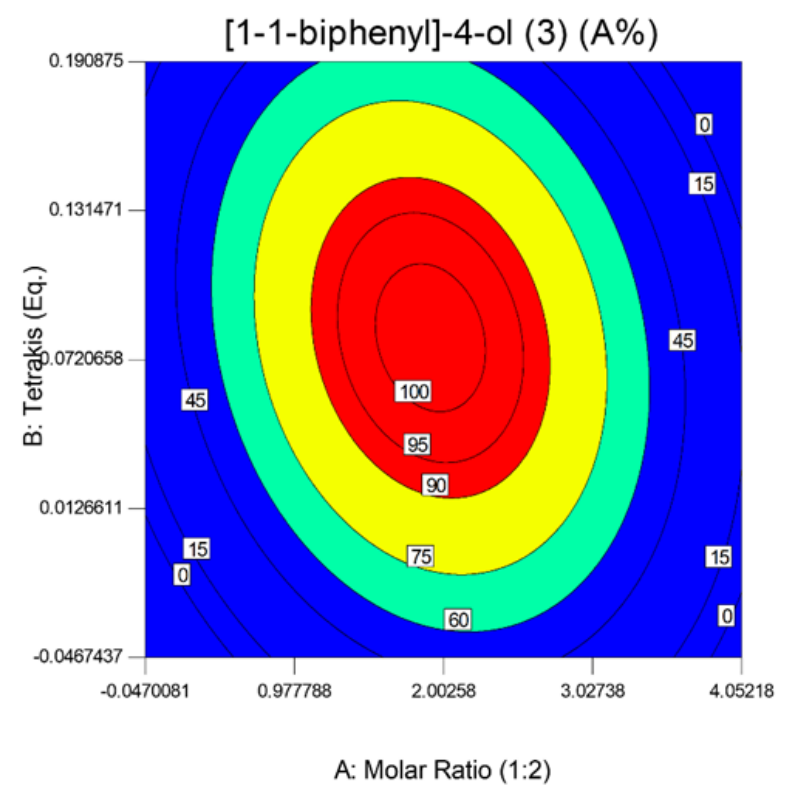

Figure 3. Effect of tetrakis and molar ratio on the [1-1-biphenyl]-4-ol in the contour plot.

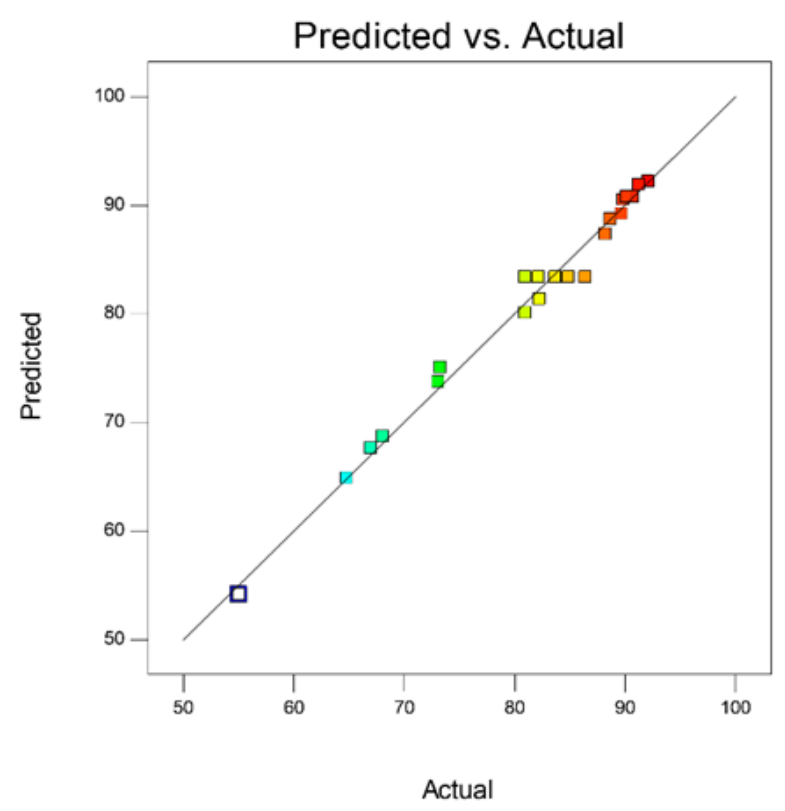

Figure 5. Relation of predicted and observed values of [1-1-biphenyl]-4-ol. high, and all other factors kept at low levels. In the case of dehydroxilated impurity (5), which was found to be in the range of 0.2 to $1.9 \mathrm{~A} \%$, when all the factors at high levels, respectively.

\subsection{Model Fitting and Optimization}

An optimization exercise was undertaken with setting a constraint to each input parameter to maximize compound [1-1-biphenyl]-4-ol (3) and to minimize (4) and (5). The experi-

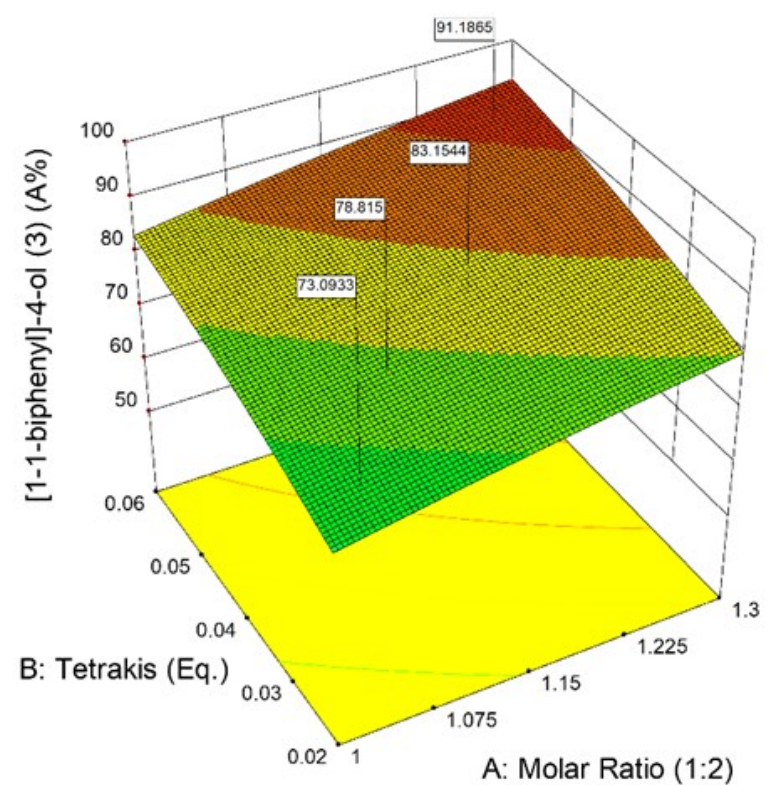

Figure 4. Effect of tetrakis and molar ratio on the [1-1-biphenyl]-4-ol in the surface plot.

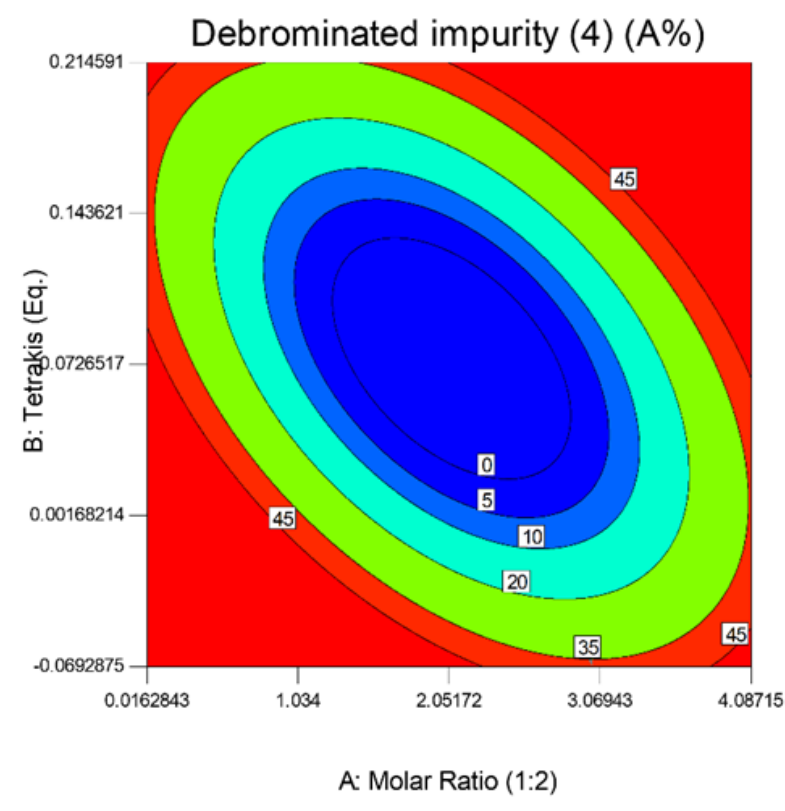

Figure 6. Effect of tetrakis and molar ratio on the debrominated impurity in the contour plot. 
mental design matrix was of the central composite design of response surface methodology, presented in Table 1 . The sequence of the experiment was randomized to minimize the effects of uncontrolled factors. The objective of the present study is to determine the optimal values of molar ratio $\left(X_{1}\right)$ of phenylboronic acid (1) and 4-bromophenol (2), catalyst tetrakis(triphenylphosphine)palladium $\left(X_{2}\right)$ equivalence, reaction temperature $\left(X_{3}\right)$, the equivalence of base tripotassium phosphate

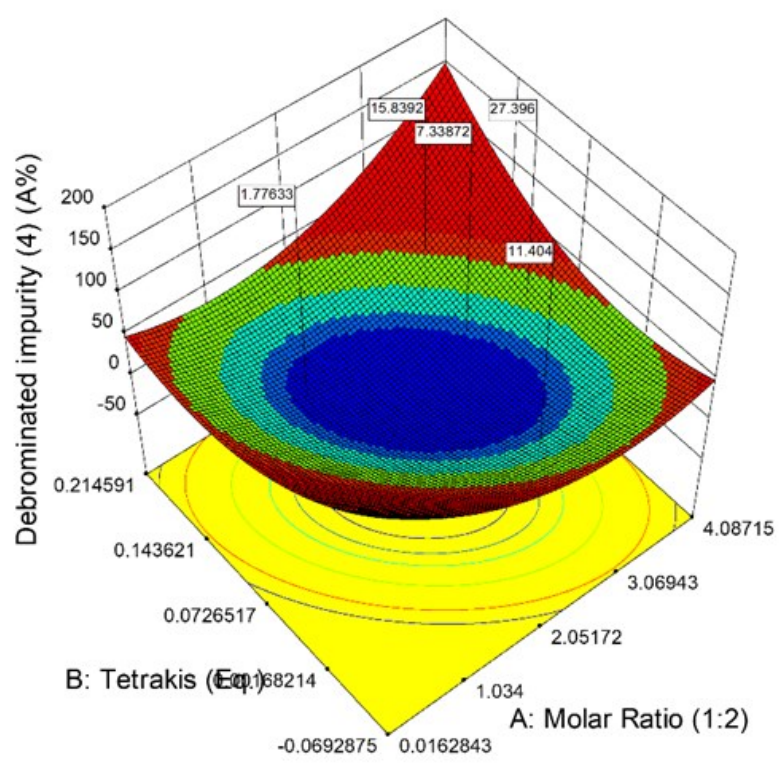

Figure 7. Effect of tetrakis and molar ratio on the debrominated impurity in the surface plot.

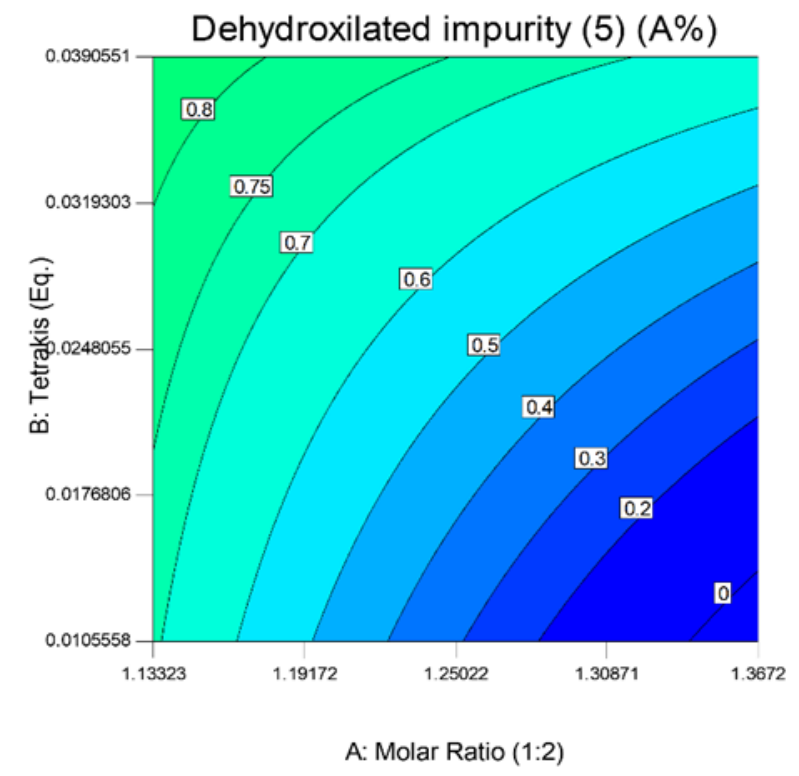

Figure 9. Effect of tetrakis and molar ratio on the dehydroxilated impurity in the contour plot.
$\left(X_{4}\right)$ to achieve maximum of [1-1-biphenyl]-4-ol (3) and to minimize impurities (4) and (5). The coefficients of the models developed in Equation (3) are estimated using multiple regression analysis techniques.

A selectivity of $87.24 \%$ [1-1-biphenyl]-4-ol (3), $10.67 \%$ debrominated impurity (4) and $0.28 \%$ dehydroxilated impurity (5) could be achieved with 1.3 molar ratio of phenylboronic acid (1) and 4-bromophenol (2), the temperature of $75.32{ }^{\circ} \mathrm{C}, 2.0$ equivalence of base tripo-

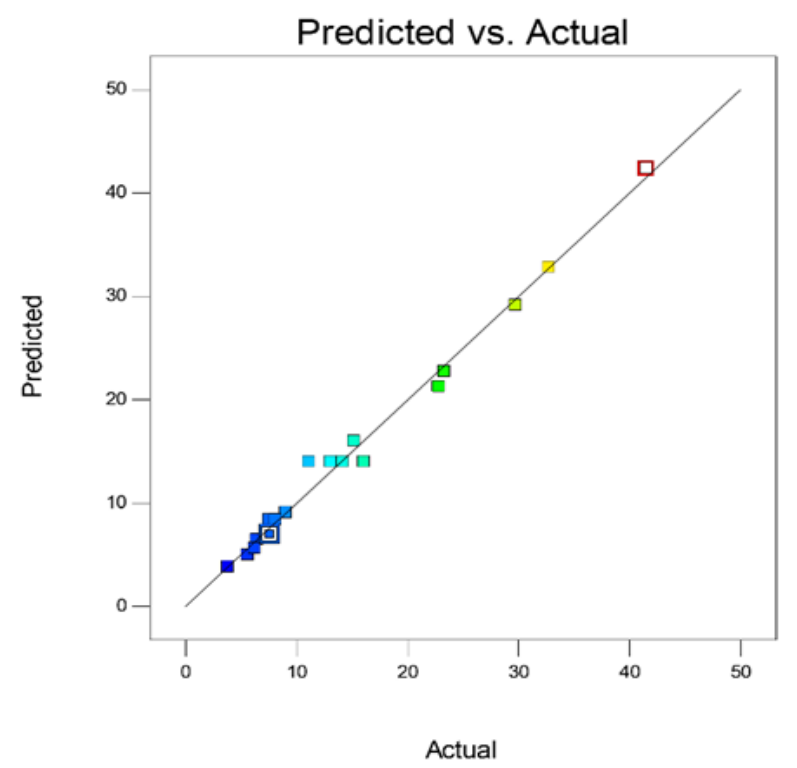

Figure 8. Relation of predicted and observed values of debrominated impurity.

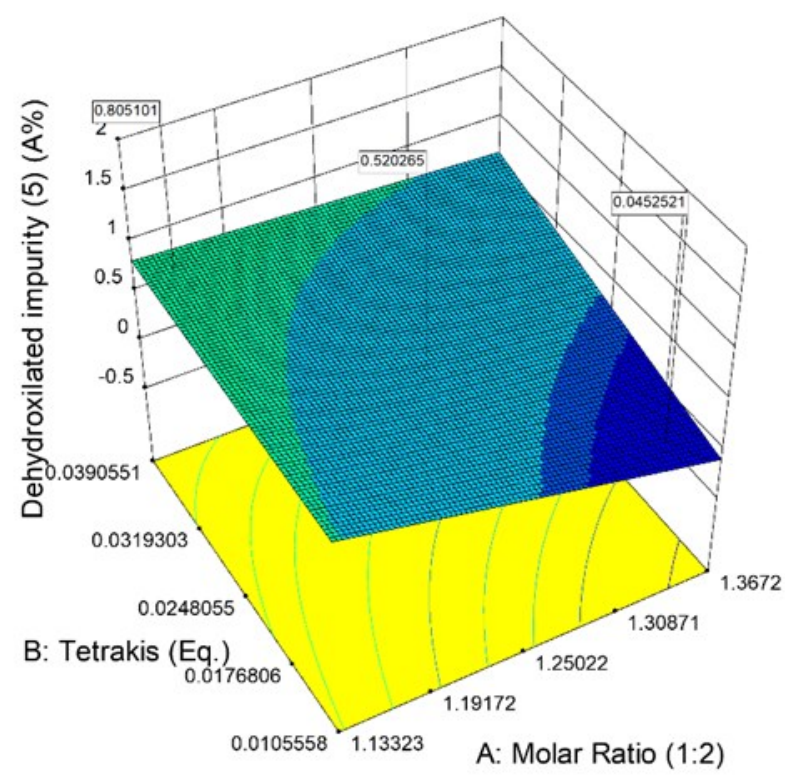

Figure 10. Effect of tetrakis and molar ratio on the dehydroxilated impurity in the surface plot. 
tassium phosphate and 0.03 equivalence of tetrakis(triphenylphosphine)palladium with the desirability of more than $87 \%$. The predicted vs actual values were in good agreement. The model equations were derived for the compound [1-1-biphenyl]-4-ol (3), debrominated impurity (4), and dehydroxilated impurity (5) and presented as Equations (4-6).

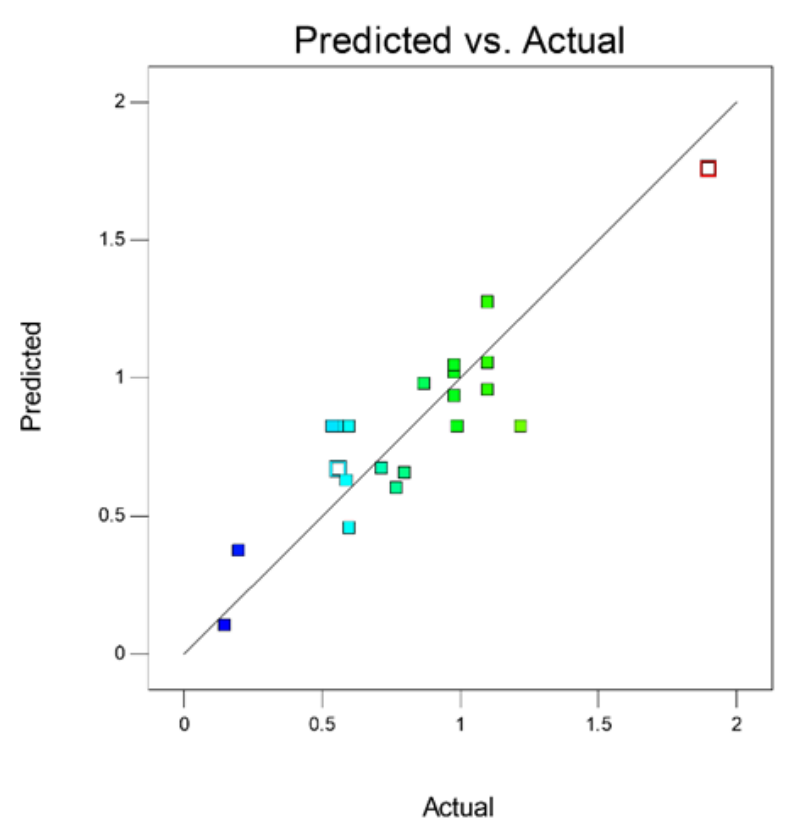

Figure 11. Relation of predicted and observed values of dehydroxilated impurity.

$$
\begin{aligned}
{[1-1} & - \text { biphenyl }]-4-o l=-64.37+121.35 X_{1} \\
& +669.172 X_{2}-5.20 X_{3}+1.03 X_{4} \\
& -87.52 X_{1} X_{2}-4.27 X_{1} X_{3}-0.366 X_{1} X_{4} \\
& +32.05 X_{2} X_{3}-0.8 X_{2} X_{4}+0.11 X_{3} X_{4} \\
& -19.42 X_{1}^{2}-3201.6 X_{2}^{2}-1.74 X_{3}^{2} \\
& -0.003 X_{4}^{2}
\end{aligned}
$$

Debrominated impurity $=153.11-106.33 X_{1}$

$-768.6 X_{2}+5.75 X_{3}-1.01 X_{4}+181.8 X_{1} X_{2}$

$+3.99 X_{1} X_{3}+0.33 X_{1} X_{4}-29.89 X_{2} X_{3}$

$+0.73 X_{2} X_{4}-0.09 X_{3} X_{4}+13.49 X_{1}^{2}$

$+2703 X_{2}^{2}+1.21 X_{3}^{2}+0.002 X_{4}^{2}$

Dehydroxilated impurity $=-5.64-1.78 X_{1}$

$$
\begin{aligned}
& -5.46 X_{2}+5.95 X_{3}-0.011 X_{4}+95.52 X_{1} X_{2} \\
& -2.81 X_{1} X_{3}+0.054 X_{1} X_{4}-35.17 X_{2} X_{3} \\
& -0.132 X_{2} X_{4}-0.031 X_{3} X_{4}
\end{aligned}
$$

The relevance of the models is judged from the determination coefficient, $\mathrm{R}^{2}$, which reveals a total variation of the observed values of activity about its mean. In this study, the regression coefficients are estimated with a satisfactory determination coefficient of $\mathrm{R}^{2}=0.98$ for the [1-1-biphenyl]-4-ol (3), 0.99 for debrominated impurity (4) and 0.79 for dehydroxilated impurity (5). The $\mathrm{R}^{2}$ value means a good agreement between the experimental and the predicted values of the fitted model. The adequacy of the empirical model was also checked by

Table 2. ANOVA study for [1-1-biphenyl]-4-ol empirical model fitting.

\begin{tabular}{lcccccc}
\hline Source & $\begin{array}{c}\text { Sum of } \\
\text { Squares }\end{array}$ & df & $\begin{array}{c}\text { Mean } \\
\text { Square }\end{array}$ & $\begin{array}{c}\mathrm{F} \\
\text { Value }\end{array}$ & $\begin{array}{c}\text { p-value } \\
\text { Prob }>\text { F }\end{array}$ & Remarks \\
\hline Model & 2120.9 & 14 & 151.49 & 32.60 & 0.00017 & significant \\
\hline $\mathrm{X}_{1}$-Molar Ratio & 140.4 & 1 & 140.43 & 30.22 & 0.00152 & \\
$\mathrm{X}_{2}$-Tetrakis & 243.6 & 1 & 243.58 & 52.42 & 0.00035 & \\
$\mathrm{X}_{3}$-Tripotassium phosphate & 241.5 & 1 & 241.45 & 51.96 & 0.00036 & \\
$\mathrm{X}_{4}$-Temperature & 294.1 & 1 & 294.09 & 63.29 & 0.00021 & \\
$\mathrm{X}_{1} \mathrm{X}_{2}$ & 0.2 & 1 & 0.23 & 0.05 & 0.83187 & \\
$\mathrm{X}_{1} \mathrm{X}_{3}$ & 0.8 & 1 & 0.82 & 0.18 & 0.68886 & \\
$\mathrm{X}_{1} \mathrm{X}_{4}$ & 4.0 & 1 & 4.00 & 0.86 & 0.38939 & \\
$\mathrm{X}_{2} \mathrm{X}_{3}$ & 0.8 & 1 & 0.82 & 0.18 & 0.68872 & \\
$\mathrm{X}_{2} \mathrm{X}_{4}$ & 0.3 & 1 & 0.34 & 0.07 & 0.79476 & \\
$\mathrm{X}_{3} \mathrm{X}_{4}$ & 10.5 & 1 & 10.48 & 2.26 & 0.18378 & \\
$\mathrm{X}_{1}{ }^{2}$ & 2.9 & 1 & 2.85 & 0.61 & 0.46306 & \\
$\mathrm{X}_{2}{ }^{2}$ & 24.5 & 1 & 24.51 & 5.27 & 0.06138 & \\
$\mathrm{X}_{3}{ }^{2}$ & 2.8 & 1 & 2.85 & 0.61 & 0.46350 & \\
$\mathrm{X}_{4}{ }^{2}$ & 24.2 & 1 & 24.24 & 5.22 & 0.06244 & \\
\hline Residual & 27.9 & 6 & 4.65 & & & \\
Lack of Fit & 9.3 & 2 & 4.64 & 1.00 & 0.44536 & not significant \\
Pure Error & 18.6 & 4 & 4.65 & & & \\
Cor Total & 2148.8 & 20 & & & & \\
\hline
\end{tabular}


analysis of variance (ANOVA) as presented in Table 2-4. In general, the calculated F-value should be greater than that of the tabulated value of the model or lower $p$-value to be considered as good fitting [40].

\section{Conclusion}

A custom-made tubular flow reactor in conjunction with multivariate DoE was effectively used to develop, model, and optimize the given Suzuki coupling reaction. The reaction profile from the flow reactor was in reasonable agree- ment with batch conditions and significant improvements to the overall reaction time and selectivity towards the desired compound [1-1biphenyl]-4-ol (3) was achieved. The Suzuki coupling reaction in batch condition would take on an average of 4 to 6 hours to complete, which was effectively accomplished in 60 to 70 minutes in this tubular reactor setup and could be operated continuously. The results showed better selectively towards compound [1-1biphenyl]-4-ol (3), with minimal impurities (4) and (5). In summary, we have found that the selectivity of compound [1-1-biphenyl]-4-ol (3)

Table 3. ANOVA study for debrominated impurity empirical model fitting.

\begin{tabular}{lcccccc}
\hline Source & $\begin{array}{c}\text { Sum of } \\
\text { Squares }\end{array}$ & df & $\begin{array}{c}\text { Mean } \\
\text { Square }\end{array}$ & $\begin{array}{c}\text { F- } \\
\text { Value }\end{array}$ & $\begin{array}{c}\text { p-value } \\
\text { Prob }>\text { F }\end{array}$ & Remarks \\
\hline Model & 2126.95 & 14 & 151.93 & 42.88 & 0.00008 & significant \\
\hline $\mathrm{X}_{1}$-Molar Ratio & 123.85 & 1 & 123.85 & 34.96 & 0.00104 & \\
$\mathrm{X}_{2}$-Tetrakis & 291.89 & 1 & 291.89 & 82.39 & 0.00010 & \\
$\mathrm{X}_{3}$-Tripotassium phosphate & 198.61 & 1 & 198.61 & 56.06 & 0.00029 & \\
$\mathrm{X}_{4}$-Temperature & 276.90 & 1 & 276.90 & 78.15 & 0.00012 & \\
$\mathrm{X}_{1} \mathrm{X}_{2}$ & 0.99 & 1 & 0.99 & 0.28 & 0.61678 & \\
$\mathrm{X}_{1} \mathrm{X}_{3}$ & 0.72 & 1 & 0.72 & 0.20 & 0.66861 & \\
$\mathrm{X}_{1} \mathrm{X}_{4}$ & 3.38 & 1 & 3.38 & 0.95 & 0.36645 & \\
$\mathrm{X}_{2} \mathrm{X}_{3}$ & 0.71 & 1 & 0.71 & 0.20 & 0.66906 & \\
$\mathrm{X}_{2} \mathrm{X}_{4}$ & 0.29 & 1 & 0.29 & 0.08 & 0.78552 & \\
$\mathrm{X}_{3} \mathrm{X}_{4}$ & 7.24 & 1 & 7.24 & 2.04 & 0.20290 & \\
$\mathrm{X}_{1}{ }^{2}$ & 1.37 & 1 & 1.37 & 0.39 & 0.55685 & \\
$\mathrm{X}_{2}{ }^{2}$ & 17.47 & 1 & 17.47 & 4.93 & 0.06815 & \\
$\mathrm{X}_{3}{ }^{2}$ & 1.38 & 1 & 1.38 & 0.39 & 0.55519 & \\
$\mathrm{X}_{4}{ }^{2}$ & 21.26 & 1 & 21.26 & 6.00 & 0.04981 & \\
\hline Residual & 21.26 & 6 & 3.54 & & & \\
Lack of Fit & 8.17 & 2 & 4.08 & 1.25 & 0.37928 & not significant \\
Pure Error & 13.09 & 4 & 3.27 & & & \\
Cor Total & 2148.21 & 20 & & & & \\
\hline
\end{tabular}

Table 4. ANOVA study for dehydroxilated impurity empirical model fitting.

\begin{tabular}{lcccccc}
\hline Source & $\begin{array}{c}\text { Sum of } \\
\text { Squares }\end{array}$ & df & $\begin{array}{c}\text { Mean } \\
\text { Square }\end{array}$ & $\begin{array}{c}\mathrm{F} \\
\text { Value }\end{array}$ & $\begin{array}{c}\text { p-value } \\
\text { Prob }>\mathrm{F}\end{array}$ & Remarks \\
\hline Model & 2.286 & 10 & 0.23 & 3.86 & 0.022 & significant \\
\hline $\mathrm{X}_{1}$-Molar Ratio & 0.048 & 1 & 0.05 & 0.81 & 0.389 & \\
$\mathrm{X}_{2}$-Tetrakis & 0.076 & 1 & 0.08 & 1.29 & 0.283 & \\
$\mathrm{X}_{3}$-Tripotassium phosphate & 0.239 & 1 & 0.24 & 4.03 & 0.072 & \\
$\mathrm{X}_{4}$-Temperature & 0.405 & 1 & 0.41 & 6.83 & 0.026 & \\
$\mathrm{X}_{1} \mathrm{X}_{2}$ & 0.272 & 1 & 0.27 & 4.59 & 0.058 & \\
$\mathrm{X}_{1} \mathrm{X}_{3}$ & 0.356 & 1 & 0.36 & 6.01 & 0.034 & \\
$\mathrm{X}_{1} \mathrm{X}_{4}$ & 0.090 & 1 & 0.09 & 1.51 & 0.247 & \\
$\mathrm{X}_{2} \mathrm{X}_{3}$ & 0.990 & 1 & 0.99 & 16.70 & 0.002 & \\
$\mathrm{X}_{2} \mathrm{X}_{4}$ & 0.009 & 1 & 0.01 & 0.16 & 0.699 & \\
$\mathrm{X}_{3} \mathrm{X}_{4}$ & 0.137 & 1 & 0.14 & 2.32 & 0.159 & \\
\hline Residual & 0.593 & 10 & 0.06 & & & \\
Lack of Fit & 0.219 & 6 & 0.04 & 0.39 & 0.854 & not significant \\
Pure Error & 0.373 & 4 & 0.09 & & & \\
Cor Total & 2.878 & 20 & & & & \\
\hline
\end{tabular}


increases with an increase in reaction temperature, catalyst tetrakis(triphenylphosphine) palladium equivalence and the molar ratio of phenylboronic acid (1) and 4-bromophenol (2) and has an inverse impact with an increase in base tripotassium phosphate equivalence. On the other side, the level of impurity (4) was found to increase when, the base tripotassium phosphate equivalence was loaded high, and all other factors kept at low levels. In the case of impurity (5), which was found to be in the range of 0.2 to $1.9 \mathrm{~A} \%$, when all the factors at high levels respectively. Apart from the numerous benefits of the continuous process, we were able to develop a model that could allow us to play around various parameters to maximize the responses across the given boundary conditions. Furthermore, we could establish statistical significance across each parameter, their weights, and their effects concerning the compound [1-1biphenyl]-4-ol (3), impurities (4), and (5). The reaction model was in good agreement with the reaction conditions. The initial practicability and development of the flow process for the Suzuki coupling were successfully demonstrated using customized tubular reactor setup, which could be further studied using precise equipment to fine-tune the process conditions for improved selectivity. The demonstrated methods could be used effectively to leverage the benefits of continuous process techniques over batch processes. We believe the process could be executed continuously without a break, readily scaled to kilogram quantities in a short period without further development.

\section{Acknowledgments}

The authors acknowledge and thank Syngene International Ltd, Bengaluru, India for the facility and financial support for this work. The authors would also like to acknowledge and thank Dr. Jegadeesh Thampi, BU-Head, Chemical Development, Syngene International Ltd, Dr. Subramanya Hegde, Dr. Satheesh Rai, and Dr. Nagaraj Chowdappa for providing adequate support, guidance, and motivation throughout the work.

\section{Supplementary Information}

The supplementary data (procedure, data analysis, and model equations, etc.) associated with this article can be found on the journal's homepage.

\section{References}

[1] Yin, L., Liebscher, J. (2007). Carbon-carbon coupling reactions catalyzed by heterogeneous palladium catalysts. Chemical Reviews, 107, 133-173.

[2] Agrofoglio, L.A., Gillaizeau, I., Saito, Y. (2003). Palladium-assisted routes to nucleosides. Chemical Reviews, 103, 1875-1916.

[3] Crawford, K.A. (2014). Direct comparison of homogeneous and heterogeneous palladium (II) catalysts for Suzuki-Miyaura crosscoupling reactions. PhD Thesis, The University of Texas at Austin, USA.

[4] Barnard, B.C. (2008). Palladium-catalyzed C-C Coupling: Then and Now. Platinum Metals Review, 52, 38-45.

[5] Johansson Seechurn, C.C., Kitching, M.O., Colacot, T.J., Snieckus, V. (20120. Palladium-catalyzed cross-coupling: a historical contextual perspective to the 2010 Nobel Prize. Angewandte Chemie International Edition, 51, 5062-5085.

[6] Len, C., Bruniaux, S., Delbecq, F., Parmar, V.S. (2017). Palladium-catalyzed SuzukiMiyaura cross-coupling in continuous flow. Catalysts, 7, 146.

[7] Paterson, I., Davies, R.D., Marquez, R. (2001). Total synthesis of the callipeltoside aglycon. Angewandte Chemie, 113, 623-627.

[8] Hiyama, T., Hatanaka, Y. (1994). Palladiumcatalyzed cross-coupling reaction of organometalloids through activation with fluoride ion. Pure and applied chemistry, 66, 1471-1478.

[9] Haswell, S.J., Watts, P. (2003). Green chemistry: synthesis in micro reactors. Green Chemistry, 5, 240-249.

[10] Wiles, C., Watts, P. (2012). Continuous flow reactors: a perspective. Green Chemistry, 14, 138-154.

[11] Newman, S.G., Jensen, K.F. (2013). The role of flow in green chemistry and engineering. Green chemistry, 15, 1456-1472.

[12] Wiles, C., Watts, P. (2014). Continuous process technology: a tool for sustainable production. Green Chemistry, 16, 55-62.

[13] Vaccaro, L., Lanari, D., Marrocchi, A., Strappaveccia, G. (2014). Flow approaches towards sustainability. Green Chemistry, 16, 3680-3704.

[14] Falß, S., Tomaiuolo, G., Perazzo, A., Hodgson, P., Yaseneva, P., Zakrzewski, J., Guido, S., Lapkin, A., Woodward, R., Meadows, R.E. (2016). A continuous process for BuchwaldHartwig amination at micro-, lab-, and mesoscale using a novel reactor con- 
cept. Organic Process Research \& Development, 20, 558-567.

[15] Suzuki, A. (2011). Cross-coupling reactions of organoboranes: an easy way to construct $\mathrm{C}-\mathrm{C}$ bonds (Nobel Lecture). Angewandte Chemie International Edition, 50, 6722-6737.

[16] Shu, W., Pellegatti, L., Oberli, M.A., Buchwald, S.L. (2011). Continuous-Flow Synthesis of Biaryls Enabled by Multistep Solid - H andling in a Lithiation/ Borylation/Suzuki-Miyaura Cross-Coupling Sequence. Angewandte Chemie, 123, 10853-10857.

[17] Kobayashi, S. (2016). Flow "fine" synthesis: high yielding and selective organic synthesis by flow methods. Chemistry-An Asian Journal, 11, 425-436.

[18] Geyer, K., Codee, J.D., Seeberger, P.H. (2006). Microreactors as tools for synthetic chemists-the chemists' round-bottomed flask of the 21st century?. Chemistry-A European Journal, 12, 8434-8442.

[19] Delville, M.M., Nieuwland, P.J., Janssen, P., Koch, K., van Hest, J.C., Rutjes, F.P. (2011). Continuous flow azide formation: Optimization and scale-up. Chemical engineering journal, 167, 556-559.

[20] Zhang, C., Zhang, J., Luo, G. (2016). Kinetic study and intensification of acetyl guaiacol nitration with nitric acid-acetic acid system in a microreactor. Journal of Flow Chemistry, 6, 309-314.

[21] Anxionnaz, Z., Cabassud, M., Gourdon, C., Tochon, P. (2008). Heat exchanger/reactors (HEX reactors): concepts, technologies: stateof-the-art. Chemical Engineering and Processing: Process Intensification, 47, 2029-2050.

[22] Koch, K., van Weerdenburg, B.J., Verkade, J.M., Nieuwland, P.J., Rutjes, F.P., van Hest, J.C. (2009). Optimizing the deprotection of the amine protecting p-methoxyphenyl group in an automated microreactor platform. Organic Process Research \& Development, 13, 1003-1006.

[23] Becker, R., van den Broek, S.B.A., Nieuwland, P.J., Koch, K., Rutjes, F.P. (2012). Optimisation and Scale-up of a-Bromination of Acetophenone in a Continuous Flow Microreactor. Journal of Flow Chemistry, 2, 87-91.

[24] Fabry, D.C., Sugiono, E., Rueping, M., Meunier, F.C. (2016). Reaction Chemistry \& Engineering. Chem. Eng., 1, 165.

[25] Nieuwland, P.J., Koch, K., van Harskamp, N., Wehrens, R., van Hest, J.C., Rutjes, F.P. (2010). Flash Chemistry Extensively Optimized: High-Temperature Swern-Moffatt Ox- idation in an Automated Microreactor Platform. Chemistry-An Asian Journal, 5, 799-805.

[26] Nagaki, A., Kim, H., Usutani, H., Matsuo, C., Yoshida, J.I. (2010). Generation and reaction of cyano-substituted aryllithium compounds using microreactors. Organic \& biomolecular chemistry, 8, 1212-1217.

[27] Hafner, A., Filipponi, P., Piccioni, L., Meisenbach, M., Schenkel, B., Venturoni, F., Sedelmeier, J. (2016). A simple scale-up strategy for organolithium chemistry in flow mode: From feasibility to kilogram quantities. Organic process research \& development, 20, 1833-1837.

[28] Gioiello, A., Mancino, V., Filipponi, P., Mostarda, S., Cerra, B. (2016). Concepts and optimization strategies of experimental design in continuous-flow processing. Journal of Flow Chemistry, 6, 167-180.

[29] Nunn, C., DiPietro, A., Hodnett, N., Sun, P., Wells, K.M. (2018). High-Throughput Automated Design of Experiment (DoE) and Kinetic Modeling to Aid in Process Development of an API. Organic Process Research \& Development, 22, 54-61.

[30] Afzal, a., Muhammad, I.A., Muhammad, Y., Hayat, K., Mansoor, u.H.S. (2013). A comparative study of alkaline hydrolysis of ethyl acetate using design of experiments. Iranian journal of chemistry and chemical engineering, 32, 33-47.

[31] Asprey, S.P., Macchietto, S. (2000). Statistical tools for optimal dynamic model building. Computers \& Chemical Engineering, 24, 1261-1267.

[32] Montgomery, D.C. (2017). Design and Analysis of Experiments. New York: John Wiley \& sons.

[33] Lazic, Z.R. (2006). Design of Experiments in Chemical Engineering: A Practical Guide. New Jersey: John Wiley \& Sons.

[34] Franceschini, G., Macchietto, S. (2008). Model-based design of experiments for parameter precision: State of the art. Chemical Engineering Science, 63, 4846-4872.

[35] Atkinson, A.C., Bogacka, B., Bogacki, M.B. (1998). D-and T-optimum designs for the kinetics of a reversible chemical reaction. Chemometrics and Intelligent Laboratory Systems, 43, 185-198.

[36] Gupta, A., Balomajumder, C. (2015). Residence time distribution study for continuous column packed with tea waste biomass. Integrated Research Advances, 2, 5-10. 
[37] Anderson, N.G. (2001). Practical use of continuous processing in developing and scaling up laboratory processes. Organic Process Research \& Development, 5, 613-621.

[38] Garcia-Serna, J., García-Verdugo, E., Hyde, J.R., Fraga-Dubreuil, J., Yan, C., Poliakoff, M., Cocero, M.J. (2007). Modelling residence time distribution in chemical reactors: A novel generalised n-laminar model: Application to supercritical $\mathrm{CO}_{2}$ and subcritical water tubular reactors. The Journal of Supercritical Fluids, 41, 82-91.
[39] Klose, F., Wolff, T., Thomas, S., SeidelMorgenstern, A. (2003). Concentration and residence time effects in packed bed membrane reactors. Catalysis today, 82, 25-40.

[40] Istadi, I., Suherman, S., Buchori, L. (2010). Optimization of reactor temperature and catalyst weight for plastic cracking to fuels using response surface methodology. Bulletin of Chemical Reaction Engineering \& Catalysis, 5 (2), 103-111, DOI:10.9767/bcrec.5.2.797.103111 